\title{
Policy Design e Múltiplas EvidênCIas: proposta analítica da dinÂMICA da politica de TRANSFORMAÇÃO DIGITAL DOS SERVIÇOS PÚBLICOS DA ADMINISTRAÇÃO PÚBLICA FEDERAL BRASILEIRA ${ }^{1,2}$
}

\author{
Pedro Lucas de Moura Palotti ${ }^{3}$ \\ Fernando de Barros Filgueiras ${ }^{4}$ \\ Maricilene Isaira Baia do Nascimento ${ }^{5}$
}

\section{INTRODUÇÃO}

As iniciativas de governo digital têm se expandido em diversos governos na atualidade. A utilização de novas tecnologias de armazenamento, gestão e integração de bases de dados de indivíduos e de novas plataformas para prestação de serviços públicos tem alterado a dinâmica de funcionamento dos governos (OECD, 2019).

Sob o discurso de maior eficiência na utilização de recursos públicos e de melhoria da satisfação dos usuários (principalmente cidadãos e empresas) com a atuação governamental, os meios virtuais e digitais têm sido mobilizados para implementar mecanismos de governo digital. Há três perspectivas principais: a primeira delas se refere à melhoria dos processos administrativos, a segunda remete à interação com os cidadãos e a terceira é a construção de interaçóes externas (Heeks, 2001).

Assim, pretende-se responder à seguinte pergunta de pesquisa: quais elementos analíticos devem ser mobilizados para se analisar o uso de evidências no design de uma política de transformação digital de serviços públicos? Ao final, tem-se como objetivo a proposição de um framework analítico para a compreensão do fenômeno de mobilização de evidências para o desenho de políticas públicas de transformação digital, a partir da experiência do governo federal brasileiro.

Esse esforço justifica-se pela incompletude e insuficiência dos modelos existentes para tratar da utilização de evidências ao longo do policy process. O modelo do ciclo de políticas públicas, por exemplo, não problematiza os instrumentos e seus subsídios em cada etapa do ciclo - inclusive a quantidade e as características das etapas do ciclo variam entre os autores. Desse modo, o framework analítico visa propor, com maior precisão, como se dá a mobilização de evidências no design de uma política pública.

A partir dessa especificidade da dinâmica das políticas públicas, a construção de um modelo analítico é importante enquanto guia na busca de respostas para a pergunta empírica levantada. Desse modo, concordamos que a abordagem de policy design é uma que contempla este propósito. Peters (2018) argumenta que o termo design é um conceito usado para descrever o processo de criação

1. DOI: http://dx.doi.org/10.38116/bapi24art7

2. Os autores agradecem os valiosos comentários e críticas dos colegas Daniel Avelino e Sivaldo Pereira da Silva. Erros e omissões restantes, contudo, são de nossa inteira responsabilidade.

3. Especialista em políticas públicas e gestão governamental (EPPGG) na Diretoria de Estudos e Políticas do Estado, das Instituições e da Democracia (Diest) do Ipea.

4. Professor na Escola de Políticas Públicas e Governo da Fundação Getulio Vargas (FGV); professor no Departamento de Ciência Política da Universidade Federal de Minas Gerais; e affiliate faculty do Ostrom Workshop on Political Theory and Policy Analysis, Indiana University. 5. Doutoranda em ciência política no Instituto de Ciência Política da Universidade de Brasília (IPOL/UnB); e pesquisadora bolsista do Programa de Pesquisa para o Desenvolvimento Nacional (PNPD) na Diest//pea. 
de uma política em resposta a um problema de política (já existente). Nesse conceito, a diferença fundamental de processos de produção de política pública diz respeito à criação de uma solução (template) que pode guiar quaisquer intervençóes.

A pesquisa foi realizada a partir da análise documental dos principais normativos e documentos vinculados à estratégia de governo digital da administração pública federal, com intuito de identificar o que informou esses normativos. A análise terá como base a perspectiva de construção institucional que balizou o processo de transformação digital em curso desde 2016.

Este artigo discorre a seguir sobre os problemas e as perspectivas da literatura internacional sobre o uso de evidências para a transformação digital dos serviços públicos. Na seção 2, são expostos os principais elementos analíticos que irão compor o framework para compreensão do policy design e sua relação com a mobilização de múltiplas evidências. Na sequência, o artigo dedica-se a esboçar o design da política de transformação dos serviços públicos de atendimento da administração pública federal brasileira, a partir da identificação de suas principais ferramentas de implementação. Por fim, são feitas algumas consideraçóes finais e é apresentada uma agenda futura de pesquisa.

\section{TRANSFORMAÇÃO DIGITAL DE SERVIÇOS PÚBLICOS E O USO DE EVIDÊNCIAS: PROBLEMA E PERSPECTIVAS}

A digitalização dos serviços públicos, com o objetivo de produzir automação dos serviços, cria novas capacidades e um processo de "plataformização" da relação entre governos e sociedade. Os governos têm seguido uma tendência crescente de "plataformização" (Dijck, Poell e Waal, 2018; Helmond, 2015). Plataformas de governo consideram as experiências de modelos de mídia social como Facebook, Youtube, Twitter, entre outras, para promover a interação entre duas ou mais partes distintas nas transaçóes governamentais. As plataformas fornecem um novo modo de comunicação entre governos e sociedade, especialmente no que diz respeito a serviços e políticas públicas, envolvendo camadas de inteligência artificial, machine learning e novos parâmetros computacionais para modificar a experiência do usuário (O’Reilly, 2010).

Basicamente, a melhoria dos serviços públicos por meio das ferramentas digitais ocorre pelo processo de digitalização e mudança nos padrôes de governança (Dunleavy e Margetts, 2013). De um lado, a digitalização de serviços públicos ocorre pela adoção de sistemas de automação. A automação de serviços públicos acontece por meio de um processo baseado em regras, em que os serviços têm os seus trâmites redesenhados para que possam ser realizados pela transação entre o usuário e a máquina. De outro lado, o processo de digitalização se dá pela adoçáo de sistemas que aumentam as capacidades de entrega dos serviços. Esta perspectiva de prestação de serviços públicos ocorre por meio da adoção de sistemas que promovam novas experiências aos usuários e melhorias contínuas por meio de machine learning (Veale e Brass, 2019).

O uso de ferramentas digitais possibilita um meio para as organizaçóes enfrentarem problemas de interrupção, concorrência e incentivos (Dunleavy e Margetts, 2013). As ferramentas digitais levaram a mudanças organizacionais decorrentes de mudanças na sociedade civil e do posicionamento político e institucional dos gestores públicos. A governança digital pode promover a reintegração de serviços em plataformas e reengenharia de processos. Ela pode criar governos mais ágeis, que respondem com rapidez e flexibilidade às mudanças no ambiente social, promovendo uma concepçáo mais holística dos serviços públicos. Por fim, a digitalização promove mudanças na entrega de serviços públicos com base nas necessidades do usuário, para gerar ganhos de produtividade (Dunleavy et al., 2006). 
Reintegração de serviço, holismo baseado em necessidades e digitalização são fatores que afetam a governança pública (Dunleavy e Margetts, 2013).

O uso de evidências no design da política de transformação digital ocorre tanto no processo de identificação dos serviços, usuários, trâmites e atores quanto na identificação de custos e experiências dos usuários que suportem o redesenho e alimentem o uso de novas ferramentas digitais. Evidências contribuem para a política de transformação digital, possibilitando identificar os problemas e justificar as soluçóes propostas, alcançando-se assim um processo de maturidade das ferramentas digitais empregadas na política.

Para seguir investindo na busca empírica sobre como diferentes atores, em diferentes processos, mobilizaram diversas fontes de evidência, a fim de se desenvolver uma política de transformação digital, a construção de um modelo analítico é central para guiar essa busca. Sendo assim, seguindo uma linha mais propositiva para orientar investigaçóes mais aprofundadas do caso de interesse, o objetivo central é construir um caminho investigativo que possa contemplar características da dinâmica da política de transformaçáo digital de serviços governamentais federais. Assim, a pergunta que mobiliza este artigo é: como analisar o uso de evidências no processo de design de uma política?

\section{POLICY DESIGN, MÚLTIPLAS EVIDÊNCIAS E PROPOSTA DE FRAMEWORK ANALÍTICO}

A utilização da terminologia e do conceito de design decorre de estudos clássicos no campo da administração pública e de políticas públicas. Um dos trabalhos pioneiros é de autoria de Simon (1996), que associa a função do designer à "ciência do artificial", dedicado a projetar como as coisas devem ser para atingir seus objetivos e cumprir sua funcionalidade.

Peters (2018) destaca que o processo de desenho de política envolve tentar integrar entendimentos do problema a ser enfrentado aos instrumentos que serão usados para a intervenção. Howlett e Mukherjee (2017) destacam também que policy design envolve a tentativa intencional de os governos ligarem instrumentos de políticas aos objetivos que buscam realizar. Envolve, além disso, compreender as vantagens e desvantagens dessas ferramentas, no intuito de aumentar a efetividade da política contemplada.

Sobre outros aspectos envolvidos, Peters (2018) acrescenta que o desenho da política está inserido em um contexto político, que chama atenção para o fato de que náo apenas a visão técnica importa no processo, mas envolve ainda: a interação de múltiplos atores; o conhecimento sobre como a área de política afetada funciona para a produção de um denominador comum; e a consideraçáo das consequências políticas da escolha da política.

Nesse processo, mudanças são desenvolvidas para responder aos balanços avaliativos da efetividade da política conhecida como situaçóes de redesenho: uma resposta do desenho atual a adaptaçóes que foram julgadas necessárias. Somado a isso, o desenho tem de considerar em quais comportamentos humanos a implementação resultará. Para isso, saber manipular símbolos e informaçóes é crucial para torná-la aceitável aos afetados pela política. Muitas das adequaçóes necessárias são referentes a aspectos dos comportamentos e dos valores humanos afetados pela política desenhada. Essas adequaçóes também são resultantes do conhecimento mais aprofundado do limite da área que deveria ser atingida pela política.

Em suma, Peters (2018) propóe um modelo de policy design que resume o processo de política em: identificar a causa de um problema (causação), estabelecer os instrumentos necessários 
(instrumentação), avaliar o desenho (avaliação) e intervir (intervenção). Desse modo, o design pode ser compreendido como a tentativa de se produzir resultados duradouros em relação a problemas comuns que atingem as coletividades. Isso implica a utilização de instrumentos de políticas públicas que possam contribuir com soluçóes sustentáveis no tempo (Capano e Woo, 2018).

Conforme já ressaltado, por envolver muitos atores, processos e instrumentos e, por consequência, diferentes interpretaçóes (técnicas e políticas), partimos do entendimento de que o desenho da política pública envolve uma multiplicidade de naturezas de evidência. Os atores mobilizam diferentes conhecimentos e informaçóes, a partir de diferentes estratégias, para sustentar uma ideia de política transformativa. Concordamos com a visão de Wesselink, Colebatch e Pearce (2014) sobre o argumento de que os participantes terão distintas, sobrepostas e conflitantes visóes acerca da natureza do problema identificado e do conjunto de conhecimentos que seráo mais apropriados para desenhar uma resposta de resolução.

Além disso, concordamos também que o uso de evidências, em suas diferentes naturezas, tem o contexto como fator central, conforme ainda defendem Wesselink, Colebatch e Pearce (2014, p. 342, traduçáo nossa): a depender do contexto, pratictioners "mobilizam o discurso e a evidência apropriada no tempo certo". Para isso, "percebem múltiplas fontes de evidência, reconhecendo o conhecimento ou julgamento prático em vez de privilegiar estudos que prometem oferecer explicações e prediçóes law-like" (idem, ibidem). Essa também é a proposta de Veselý, Ochrana e Nekola (2018, p. 223), que seguem uma corrente sobre os estudos de evidência na política pública os quais mostram que os policymakers desenham a política a partir de uma ampla variedade de informação e conhecimento e, portanto, "suas interpretaçóes do que conta como evidência útil e relevante é simplesmente diferente dos proponentes do [evidence-based policy] EBP”. Acrescentam ainda o pressuposto de que o uso de evidência é um processo dinâmico, emaranhado em um amplo processo de design da política pública (Veselý, Ochrana e Nekola, 2018, p. 224).

Nessa oportunidade de reconhecer que a burocracia estatal mobiliza diferentes evidências e discursos estratégicos de maneira relacionada, examinar como atores usam a evidência em suas narrativas é uma estratégia metodológica de muita valia. A exemplo da proposta de Schlaufer (2018, p. 90), concordamos que "O uso narrativo de evidência é definido pelos diferentes papéis que [um tipo] de evidência desempenha no enredo de uma narrativa”. Contudo, evidências são instrumentalizadas em diversas fontes para construir uma narrativa sobre a política.

Mais especificamente, of framework analítico que propomos construir vai ao encontro da proposta de Head (2008). A defesa do autor parte do entendimento de que as decisóes sobre políticas são oriundas de um contexto político, com julgamentos e debates, mais que de uma simples dedução de análises empíricas. Debates e análises no âmbito da produção de política pública envolvem intercâmbios entre fatos, normas e açóes desejáveis, em que a evidência ganha uma natureza diversa e contestável.

Sendo assim, Head (2008, p. 4, tradução nossa) argumenta que "soluçôes técnicas simples de especialistas não estão disponíveis ou são impraticáveis. Nessas circunstâncias, pode surgir uma abordagem 'negociada' e 'relacional' para a solução de problemas”. Uma abordagem relacional para essa discussão reconhece a dimensáo da negociação em que "redes e parcerias trazem para a mesa de negociação uma diversidade de 'evidências' das partes interessadas, ou seja, informações, interpretaçóes e prioridades relevantes” (Head, 2008, p. 4, traduçáo nossa). 
Head (2008), portanto, propóe três tipos importantes de conhecimento (aos quais suas visões de "evidência" correspondem): conhecimento político; conhecimento científico (baseado em pesquisa); e conhecimento prático-profissional. No campo do conhecimento político, atores políticos mobilizam análises e julgamentos para desenvolver melhores estratégias, táticas, persuasão, comunicaçóes-chave, bem como moldar e responder a questóes de accountability, entre outras. No campo do conhecimento científico, produtos de análises sistemáticas são mobilizados para explicar causas inter-relacionadas para explicar condiçóes e prediçóes. Por fim, no campo do conhecimento prático-profissional, experiências da prática profissional são oriundas de comunidades de práticas profissionais e de conhecimentos organizacionais associados com gerenciamento de implementação de política.

A utilização de evidências é um insumo relevante para o design das políticas, por vezes assumindo um papel instrumental, conceitual ou simbólico (Amara, Ouimet e Landry, 2004). A mobilização, captação, interpretação e utilização de evidências, em suas diferentes perspectivas, perpassam a construçáo de capacidades analíticas, gerenciais e políticas dos gestores governamentais. Essas capacidades serão então aplicadas à construção de soluçóes de políticas públicas que possam se tornar efetivas (Mukherjee e Bali, 2019).

A figura 1 tenta traduzir, em síntese, os elementos teórico-conceituais que guiarão a pesquisa em torno da relação entre a busca por soluções e o uso de diferentes fontes de evidência. A disposição dos elementos na figura é uma tentativa de representar processualmente a possível configuração da dinâmica da política de transformação digital dos serviços públicos federais no Brasil. Essa política é uma tendência mundial que, no Brasil, também foi pautada no discurso sobre a redução de custos (oportunidade fiscal) para o Estado e no discurso sobre o aumento da acessibilidade do cidadão aos serviços prestados por ele (oportunidade social) (Heckert, 2018). Argumentamos que o histórico tendente de crise fiscal intensificou a busca por esses discursos justificadores como uma janela de oportunidade para acelerar o desenvolvimento dessa política.

Do ponto de vista social, uma evidência que talvez represente o discurso da acessibilidade ao cidadão é o marco normativo do governo eletrônico no país, estabelecido pelo Decreto oㅡ 6.932, de agosto de 2009, conhecido como Decreto do Cidadáo. O artigo 1ํo enumerava as diretrizes que órgãos e entidades do Poder Executivo deveriam observar para guiar suas relaçóes com o cidadão. Entre tais diretrizes, estava a aplicação de ferramentais digitais para simplificar os processos e procedimentos de atendimento. No Brasil, a política de transformação digital dos serviços públicos foi estabelecida basicamente por atos normativos do Poder Executivo central, por meio de decretos. Trata-se de um indicador de que é uma política mobilizada, desenhada, intensificada no âmbito da própria burocracia estatal, com um desenho longevo e incremental.

Apesar de a política de transformação digital no Brasil ser desenvolvida no seio da burocracia estatal, ainda assim é uma política interseccionada pelos problemas públicos de natureza política e administrativa, e isso implica que múltiplos atores necessariamente participam do processo. Heckert (2018) descreve que, no Brasil, o programa Plataforma de Cidadania Digital envolveu vários stakeholders - entre eles, dirigentes dos órgãos, servidores dos órgãos, fornecedores e o núcleo orçamentário-financeiro do governo. Pelo fato de distintos atores afetados e idealizadores da política estarem envolvidos no processo de desenho, propomos que diferentes evidências foram mobilizadas, cada uma estabelecendo sua própria narrativa (com intenção argumentativa) sobre a política proposta. 


\section{FIGURA 1}

Proposta gráfica da relação dos elementos teórico-conceituais (policy design e múltiplas evidências) na política de transformação digital dos serviços públicos federais no Brasil

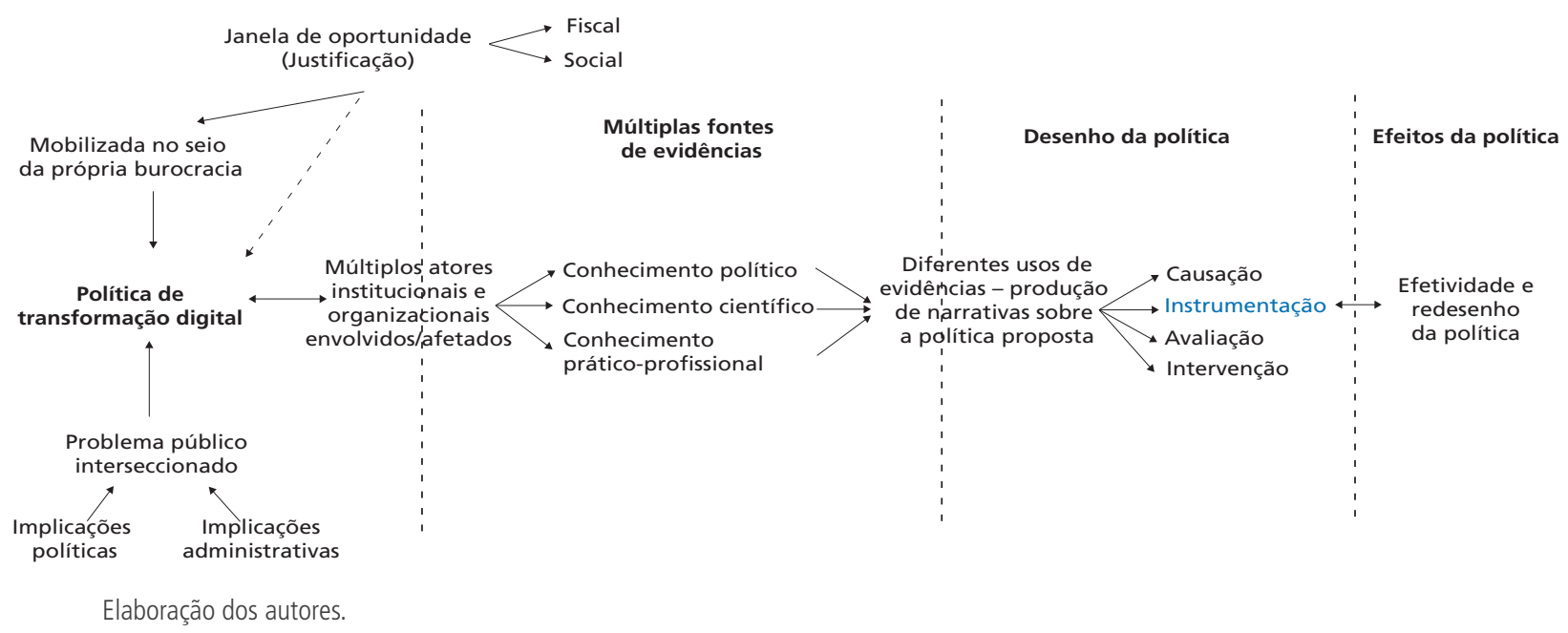

Conforme representado na figura 1, esses diferentes atores, em suas diferentes mobilizaçóes de evidências, podem fazer parte de todas as fases do desenho da política. No entanto, uma fase crucial, que vivifica e torna ativa a participação desses diferentes atores, diz respeito à fase de instrumentaçáo, parte do processo que indicará a efetividade ou náo da política e seus futuros redesenhos, em busca de novas combinaçóes de instrumentos. A dimensão da efetividade de fato é posta como um elemento central para a compreensão do design. Nas palavras de Peters et al. (2018, p. 5), "[um] objetivo central - na verdade, o objetivo central - do design de políticas públicas é a 'efetividade”, a qual "serve como o fundamento básico de qualquer design, sobre o qual outros objetivos como eficiência e equidade se baseiam”.

\section{INSTRUMENTAÇÃO E USO DE EVIDÊNCIAS PARA O DESIGN DA TRANSFORMAÇÃO DOS SERVIÇOS PÚBLICOS DE ATENDIMENTO DA ADMINISTRAÇÃO PÚBLICA FEDERAL BRASILEIRA}

No caso brasileiro, a construção de uma política pública de governo digital remonta ao início dos anos 2000, com a criação de uma política definida na época como e-gov e com a organizaçáo de um Comitê Executivo de Governo Eletrônico (CEGE). Os anos seguintes reúnem esforços de compartilhamento de infraestrutura de acesso, certificação digital organizada sobre governo digital, modelo de acessibilidade de governo eletrônico (eMAG), portal de inclusão digital, modelo de software público, entre outras iniciativas de construção institucional para fomento da governança digital (Filgueiras, Cireno e Palotti, 2019).

Entre 2016 e 2020, vários decretos presidenciais e uma lei deram uma nova institucionalidade ao processo incremental de constituição de governo digital, modificando o desenho da governança digital. Foi instituída uma nova Política de Governança Digital (Decreto no 8.638/2016, substituído pelo Decreto no 10.332/2020), uma Política de Dados Abertos para o Poder Executivo (Decreto no 8.777/2016), uma regulamentação do compartilhamento de bases de dados na administração pública federal (Decreto no 8.789/2016, posteriormente substituído pelo Decreto no 10.046/2019) e a instituição da Plataforma de Cidadania Digital (Decreto no 8.936/2016), convertida em 2019 na plataforma gov.br (Decreto n⿳⺈ 9.756/2019). Houve também a definição acerca da participação, 
proteção e defesa dos direitos dos usuários dos serviços públicos da administração federal (Lei n⿳0 13.460/2017, regulamentada pelos Decretos no 9.094/2017 e no 9.723/2019). É importante destacar o esforço complementar de elaboração do documento norteador Estratégia Brasileira para a Transformação Digital: E-Digital, a cargo do Ministério da Ciência, Tecnologia, Inovaçóes e Comunicaçôes (Decreto no 9.584/2018, substituído pelo Decreto nํㅜ 10.332/2020).

É nesse contexto que se institui o design de uma política pública de governança digital, com ênfase na transformação digital dos serviços públicos prestados pelo governo federal e posterior rede com Unidades da Federação. O mandato para a construção da plataforma de serviços públicos foi dado, até 2018, ao Ministério do Planejamento, Desenvolvimento e Gestão e, após o processo de transição de governo, à Secretaria de Desburocratização e Governo Digital do Ministério da Economia. Todo esse processo foi coordenado pela Casa Civil da Presidência da República, estando hoje, a partir do Decreto no 10.332/2020, a cargo da Secretaria-Geral da Presidência da República.

Esta política de transformação digital tem o objetivo de promover a automação incremental dos serviços públicos, especialmente aqueles que envolvam transaçóes entre o governo e cidadáos ou empresas. O desenho dessa política tem o objetivo de mobilizar um conjunto de ferramentas digitais para modificar a estrutura da governança dos serviços públicos. De um lado, automatizar os trâmites entre o governo e os usuários de serviços públicos, fazendo com que as transaçóes ocorram gradativamente sem interferência humana. De outro lado, promover melhorias na experiência do usuário, de maneira a aumentar as capacidades de entrega dos serviços por meio da aplicaçáo de ferramentas digitais.

O caso da política de transformação digital de serviços públicos no Brasil é um tipo de política que segue um processo diferente do já convencionado na literatura (ciclo de política pública). Partimos do pressuposto de que uma política desse tipo é mobilizada (e/ou intensificada) no seio da própria burocracia estatal, em resposta a um problema público identificado em políticas públicas já existentes, em que tal identificação e posterior proposição de solução, na realidade, são motivadas por um contexto existente de conjuntura crítica. Além disso, uma característica importante desse tipo de política diz respeito à necessidade de solução "centralizada" ou de centralização, um tipo que implica problematizar a autonomia e os modos de trabalho dos atores institucionais afetados pela mudança. Partimos também do pressuposto de que, para que essa política transformativa e "deslocativa" fosse possível, diferentes naturezas de evidências foram mobilizadas para convencer e justificar a necessidade de mudança.

O quadro 1 realiza uma síntese dos principais normativos, seus instrumentos e as evidências mobilizadas explicitamente para nortear a transformação digital dos serviços públicos de atendimento.

A plataforma gov.br pretende transformar a estrutura dos serviços públicos por um desenho de política que promova automação e aumento das capacidades e novas experiências do usuário. Para a transformação digital, são previstas seis fases principais, com as respectivas ferramentas: i) questione (identificação, priorização, levantamento de custos); ii) personalize (mapeamento dos atores, perspectivas e jornadas dos usuários); iii) reinvente (redesenho dos trâmites, prototipação e testagem); iv) facilite (simplificação); v) integre (integração com a Plataforma de Autenticação Digital do Cidadão - BrasilCidadão); e vi) comunique (comunicação das mudanças, com simplificação do vocabulário técnico) (Heckert, 2018). A escolha das ferramentas e o design da política de transformação digital mobilizaram evidências múltiplas para construir uma narrativa que justifique 
o processo de mudança dos serviços públicos, com especial enfoque nos benefícios da automação para o enfrentamento da crise fiscal do Estado e maior agilidade do governo para prestar serviços e ser coerente com os interesses dos usuários. Esta narrativa baseada em evidências possibilitou um processo de mudança calcado na experiência do usuário. Resta analisar, nesse design da política de transformação digital, as evidências sobre a qualidade dos serviços públicos digitais, de maneira a se realimentar o processo da política e construir aprendizado organizacional mais sólido.

QUADRO 1

Normativos, instrumentos e evidências para a transformação digital

\begin{tabular}{|c|c|c|}
\hline $\begin{array}{c}\text { Normativos ou } \\
\text { documentos norteadores }\end{array}$ & Instrumentos previstos & $\begin{array}{l}\text { Evidências mobilizadas pela proposta } \\
\text { ou com produção prevista pela proposta }\end{array}$ \\
\hline $\begin{array}{l}\text { Decreto } n^{0} 8.638 / 2016 \text {, substituído } \\
\text { pelo Decreto no } 10.332 / 2020\end{array}$ & $\begin{array}{l}\text { Política de Governança Digital, cujo desdobramento faz } \\
\text { referência à/ao: } \\
\text { a) Estratégia Brasileira de Governança Digital; } \\
\text { b) omitê de Governança Digital nos órgãos e nas } \\
\text { entidades da administração pública federal; e } \\
\text { c) Rede Gov.Br. }\end{array}$ & $\begin{array}{l}\text { Remete a normativos anteriores que instituíam } \\
\text { o CEGE e seus comitês técnicos, fazendo a } \\
\text { revogação dessa estrutura anterior. }\end{array}$ \\
\hline Decreto no 8.777/2016 & $\begin{array}{l}\text { Política de Dados Abertos do Poder Executivo federal, } \\
\text { estabelecendo que: } \\
\text { a) a coordenação da política será feita pela CGU, } 1 \text { por } \\
\text { meio da Infraestrutura Nacional de Dados Abertos (Inda); e } \\
\text { b) a execução se dará por meio do Plano de Dados Abertos } \\
\text { de cada órgão ou entidade. }\end{array}$ & $\begin{array}{l}\text { Enumera um conjunto de bases de dados de } \\
\text { interesse público para priorização da Política de } \\
\text { Dados Abertos. }\end{array}$ \\
\hline $\begin{array}{l}\text { Decreto } n^{0} \text { 8.789/2016 e } \\
\text { Decreto no 10.046/2019 }\end{array}$ & $\begin{array}{l}\text { Estabelece o compartilhamento de bases de dados na } \\
\text { administração pública federal: } \\
\text { a) níveis de compartilhamento; } \\
\text { b) Cadastro Base do CidadãO; e } \\
\text { c) Comitê Central de Governança de Dados. }\end{array}$ & $\begin{array}{l}\text { Prevê a criação de catálogo das bases sobre } \\
\text { a gestão de cada órgão ou entidade federal, } \\
\text { informando os compartilhamentos vigentes. }\end{array}$ \\
\hline Decreto no 8.936/2016 & $\begin{array}{l}\text { Plataforma de Cidadania Digital, composta por: } \\
\text { a) portal de serviços do governo federal; } \\
\text { b) mecanismo de acesso digital único do usuário a } \\
\text { serviços públicos; } \\
\text { c) ferramenta de solicitação e acompanhamento de } \\
\text { serviços públicos; } \\
\text { d) painel de monitoramento do desempenho dos serviços } \\
\text { públicos; e } \\
\text { e) Comitê Gestor da Plataforma de Cidadania Digital. }\end{array}$ & $\begin{array}{l}\text { Cadastramento e atualização de informações } \\
\text { sobre os serviços públicos no portal de serviços } \\
\text { do governo federal, pelo qual decorreu o } \\
\text { comissionamento da pesquisa Censo dos } \\
\text { Serviços Públicos de Atendimento do Governo } \\
\text { Federal (Heckert, 2018). }\end{array}$ \\
\hline Decreto no 9.756/2019 & $\begin{array}{l}\text { Portal único gov.br, de forma a: } \\
\text { a) migrar os conteúdos de seus portais na internet para o } \\
\text { portal único; e } \\
\text { b) desativar os endereços de sítios eletrônicos existentes. }\end{array}$ & $\begin{array}{l}\text { Benchmarking de outras experiências } \\
\text { internacionais coordenadas pela OCDE. }\end{array}$ \\
\hline $\begin{array}{l}\text { Lei no } 13.460 / 2017 \text { regulamentada } \\
\text { pelos Decretos no } 9.094 / 2017 \\
\text { e ํo } 9.723 / 2019\end{array}$ & $\begin{array}{l}\text { Normas básicas para participação, proteção e defesa de } \\
\text { direitos do usuário de serviços públicos: } \\
\text { a) quadro geral de serviços; } \\
\text { b) Carta de Serviços do Usuário; } \\
\text { c) conselhos de usuários; e } \\
\text { d) solicitação de simplificação. }\end{array}$ & $\begin{array}{l}\text { Órgãos e entidades devem avaliar os } \\
\text { serviços prestados quanto a: i) satisfação do } \\
\text { usuário com o serviço prestado; ii) qualidade } \\
\text { do atendimento prestado ao usuário; } \\
\text { iii) cumprimento dos compromissos e prazos } \\
\text { definidos para a prestação dos serviços; } \\
\text { iv) quantidade de manifestações de usuários; } \\
\text { e v) medidas adotadas pela administração } \\
\text { pública para melhoria e aperfeiçoamento da } \\
\text { prestação do serviço. } \\
\text { Ranking das entidades com maior incidência de } \\
\text { reclamação dos usuários e com melhor avaliação. }\end{array}$ \\
\hline
\end{tabular}


(Continuação)

\begin{tabular}{|c|c|c|}
\hline $\begin{array}{c}\text { Normativos ou } \\
\text { documentos norteadores }\end{array}$ & Instrumentos previstos & $\begin{array}{l}\text { Evidências mobilizadas pela proposta } \\
\text { ou com produção prevista pela proposta }\end{array}$ \\
\hline $\begin{array}{l}\text { E-Digital: estratégia brasileira para a } \\
\text { transformação digital } \\
\text { (Decreto no } 9.584 / 2018 \text {, substituído } \\
\text { pelo Decreto no } 10.332 / 2020 \text { ) }\end{array}$ & $\begin{array}{l}\text { Proposta de dois grandes eixos temáticos: } \\
\text { a) habilitadores (infraestrutura e acesso às TICs; }{ }^{2} \text { pesquisa, } \\
\text { desenvolvimento e inovação; confiança no ambiente } \\
\text { digital; educação e capacitação profissional; dimensão } \\
\text { internacional); e } \\
\text { b) transformação digital (economia, cidadania e governo). }\end{array}$ & $\begin{array}{l}\text { Mobilização de cinco subgrupos formados } \\
\text { por servidores públicos envolvidos com essa } \\
\text { temática; consulta focada em um grupo de } \\
130 \text { especialistas; reuniões setoriais - iniciativa } \\
\text { privada, organizações não governamentais e } \\
\text { entidades governamentais; consulta pública; e } \\
\text { citação de literatura nacional e internacional } \\
\text { sobre o tema - estudos de caso, relatório de } \\
\text { organismos internacionais etc. }\end{array}$ \\
\hline
\end{tabular}

Elaboração dos autores.

Notas: ${ }^{1}$ CGU - Controladoria-Geral da União.

${ }^{2}$ TICs - Tecnologias de Informação e Comunicação.

${ }^{3}$ OCDE - Organização para a Cooperação e Desenvolvimento Econômico.

\section{CONCLUSÃO}

O uso de tecnologias digitais que podem melhorar a governança deve se concentrar em ferramentas para implementar políticas e serviços públicos (Salamon, 2001). O conceito de serviço público supóe a estruturação de serviços implementados pelos governos para beneficiar a sociedade e garantir direitos. A agenda da transformação digital de serviços públicos tem sido incentivada pela OCDE, que criou parâmetros e princípios para a transferência de inovação no processo de serviços públicos.

Este artigo teve como intuito apresentar uma proposta de framework analítico sobre design de políticas públicas, enfatizando a dimensão da mobilização de múltiplas evidências e sua interconexão com as ferramentas de implementação de políticas públicas. Esboçou-se o processo em curso de transformação digital no âmbito do governo federal brasileiro, a partir da análise das normativas e de documentos sobre essa política. Como agenda futura de pesquisa, almeja-se o aprofundamento do entendimento sobre o processo de transformação digital. Uma das possibilidades de agenda de pesquisa é a compreensão da lógica da transformação digital de alguns serviços a partir de métodos qualitativos, como entrevistas semiestruturadas, que permitam a exploraçáo mais detalhada dos processos de implementação e de mobilização explícita ou implícita do uso de evidências no policy making.

\section{REFERÊNCIAS}

AMARA, N.; OUIMET, M.; LANDRY, R. New evidence on instrumental, conceptual, and symbolic utilization of university research in government agencies. Science Communication, v. 26, n. 1, p. 75-106, 2004.

CAPANO, G.; WOO, J. J. Designing policy robustness: outputs and processes. Policy and Society, v. 37, n. 4, p. 422-440, 2018.

DIJCK, J. V.; POELL, T.; WAAL, M. de. The platform society: public values in a connective world. Oxford: Oxford University Press, 2018. 
DUNLEAVY, P. et al. New public management is dead - long live digital-era governance. Journal of Public Administration Research and Theory, v. 16, n. 3, p. 467-494, 2006. Disponível em: <https://doi.org/10.1093/jopart/mui057>.

DUNLEAVY, P.; MARGETTS, H. The second wave of digital-era governance: a quasi-paradigm for government on the web. Philosophical Transactions of the Real Society, v. 371, n. 1.987, p. 1-17, 2013. Disponível em: <https://doi.org/10.1098/rsta.2012.0382>.

FILGUEIRAS, F.; CIRENO, F.; PALOTTI, P. Digital transformation and public service delivery in Brazil. Latin American Policy, v. 10, n. 2, p. 195-219, 2019. Disponível em: <https://doi. org/10.1111/lamp.12169>.

HEAD, B. W. Three lenses of evidence: based policy. The Australian Journal of Public Administration, v. 67, n. 1, p. 1-11, 2008.

HECKERT, C. R. Plataforma de Cidadania Digital. [s.l.]: Enap, 2018. p. 1-14. Disponível em: $<$ http://repositorio.enap.gov.br/handle/1/3457>.

HEEKS, R. Understanding e-governance for development. Manchester: University of Manchester Press, 2001.

HELMOND, A. The platformization of the web: making web data platform ready. Social Media + Society, v. 1, n. 2, p. 1-11, 2015. Disponível em: <https://doi.org/10.1177/2056305115603080>.

HOWLETT, M.; MUKHERJEE, I. Policy design: from tools to patches. Canadian Public Administration, v. 60, n. 1, p. 140-144, Mar. 2017.

OECD - ORGANISATION FOR ECONOMIC CO-OPERATION AND DEVELOPMENT. The innovation system of the public service in Brazil. Paris: OECD Publishing, 2019. Disponível em: <https://doi.org/10.1787/a1b203de-en>.

O'REILLY, T. Government as platform. In: LAHTROP, D.; RUMA, L. (Ed.). Open government: collaboration, transparency, and participation in practice. Sebastopol: O'Reilly Media, 2010.

PETERS, B. G. The logic of policy design. In: PETERS, B. G. Policy problems and policy design: new horizons in public policy. Edward Elgar: Cheltenham, 2018. p. 1-34.

PETERS, B. G. et al. Designing for policy effectiveness. Cambridge: Cambridge University Press, 2018.

SALAMON, L. The new governance and the tolls of public action: an introduction. Fordham Urban Law Journal, v. 28, n. 5, p. 1.611-1.674, 2001. Disponível em: <https://ir.lawnet.fordham.edu/ ulj/vol28/iss5/4>.

SCHLAUfER, C. The narrative uses of evidence. The Policy Studies Journal, v. 46, n. 1, p. 90-118, 2018.

SIMON, H. The sciences of the artificial. 3d ed. Cambridge: MIT Press, 1996.

UKHERJEE, I.; BALI, A. S. Policy effectiveness and capacity: two sides of the design coin. Policy Design and Practice, v. 2, n. 2, p. 103-114, 2019. 
VEALE, M.; BRASS, I. Administration by algorithm? Public management meets public sector machine learning. In: YEUNG, K.; LODGE, M. (Ed.). Algorithm regulation. Oxford: Oxford University Press, 2019.

VESELÝ, A.; OCHRANA, F; NEKOLA, M. When evidence is not taken for granted: the use and perception of "evidence" in the Czech republic ministries. NISPAcee Journal of Public Administration and Policy, v. 11, n. 2, p. 219-234, Dec. 2018.

WESSELINK, A.; COLEBATCH, H.; PEARCE, W. Evidence and policy: discourses, meanings and practices. Policy Sciences, v. 47, p. 339-344, 2014. Disponível em: <https://doi.org/10.1007/ s11077-014-9209-2>.

\section{BIBLIOGRAFIA COMPLEMENTAR}

EARLEY, S. The digital transformation: staying competitive. IT Professional, v. 16, n. 2, p. 58-60, 2014. Disponível em: <https://ieeexplore.ieee.org/document/6798637>. 
